\title{
Hipovitaminose A em recém-nascidos em duas maternidades públicas no Rio de J aneiro, Brasil
}

\author{
Hypovitaminosis $A$ in neonates from \\ public hospitals in Rio de Janeiro, Brazil
}

Rejane Andréa Ramalho 1

Luiz Antonio dos Anjos 2,3

Hernando Flores 4

\footnotetext{
1 Departamento de Nutrição e Dietética, Instituto de Nutrição Josué de Castro, Centro de Ciências da Saúde Universidade Federal do Rio de Janeiro, bloco J,

20 andar, Il ha do Fundão, Rio de Janeiro, RJ 21944-970, Brasil. aramalho@rionet.com.br

2 Laboratório de Avaliação Nutricional e Funcional, Departamento de Nutrição Social, Faculdade de Nutrição, Universidade Federal Fluminense. Rua São Paulo 30, 4o andar, Centro, Niterói, RJ 24015-110 Brasil.

3 Centro de Estudos da Saúde do Trabalhador e Ecologia Humana, Escola Nacional de Saúde Pública, Fundação Oswaldo Cruz.

Rua Leopoldo Bulhões 1480, Manguinhos, Rio de Janeiro, RJ, 21041-210, Brasil. anjos@ensp.fiocruz.br

4 Departamento de Nutrição, Universidade Federal de Pernambuco.

Cidade Universitária, Recife, PE, 50670-901, Brasil.
}

Abstract Vitamin A status (umbilical cord retinol levels) of 253 newborns in two public hospitals of Rio de Janeiro showed a high prevalence (55.7\%) of deficiency (retinol levels bel ow 1.05 $\mu \mathrm{mol} / \mathrm{l}$ ). This rate of vitamin A deficiency (VAD) was independent of other nutritional and anthropometric parameters, such as low birth weight or small for gestational age. These data suggest that newborns can be as vulnerable to VAD as other population groups consi dered at high risk. They also suggest that special attention should be paid to this group, actually the most vulnerable to the harmful effects of VAD. Hypovitaminosis A should be among the first diagnostic hypotheses when an infant presents with an abnormality.

Key words Vitamin A; Vitamin A Deficiency; Newborn, Infant; Nutritional Status

Resumo O estado nutricional de vitamina A foi avaliado, através dos níveis de retinol no sangue do cordão umbilical, em 253 recém-nasci dos assi sti dos em duas maternidades públicas do Muni cípio do Rio de Janeiro. Independentemente da idade gestacional e do peso ao nascer, a prevalência de valores baixos de retinol $(<1,05 \mu \mathrm{mol} / \mathrm{l})$ foi el evada $(55,7 \%)$. Em crianças com baixo peso, essa prevalência chegou a 68,7\%. Confirmando uma série de observações de outros autores, não se observou qualquer associação entre estado nutricional, aval iado antropometricamente, e níveis de retinol. Os resultados mostram que, na amostra investigada, a prevalência de hipovitami nose A nos recém-nasci dos é comparável às cifras que se encontram nas regiões mais pobres do mundo, e sugerem a necessi dade de se prestar especial atenção a esse grupo populacional por ser, entre os grupos de risco, o mais vulnerável aos efei tos del etérios da carência marginal de vitamina $A$, em razão do rápido cresci mento nos primei ros meses de vida.

Palavras-chave Vitamina A; Deficiência deVitamina A; Recém-Nascido; Estado Nutricional 


\section{Introdução}

A vitamina A é um nutriente essencial para muitos processos metabólicos, como a diferenciação celular, a visão, a integridade do sistema imunológico e a manutenção e renovação de epitélios, sendo de especial importância durante o crescimento e desenvolvimento (gestação, período neonatal e infância) (Underwood, 1994; WHO, 1996).

Em gestantes, tem sido observada uma tendência de diminuição dos níveis de retinol sérico, especialmente no último trimestre da gestação (Rondo et al., 1995). Por outro lado, as reservas de vitamina A do feto são baixas, por causa da seletiva barreira placentária para a passagem dessa vitamina para o feto, provavelmente para evitar efeitos teratogênicos (GebreMedhin \& Vahlquist, 1984; Olson, 1987), causando, assim, baixa reserva hepática de vitamina $A$ no recém-nascido, independentemente da ingestão materna (Gardner \& Ross, 1993).

Após o nascimento, os estoques fetais tendem a aumentar rapidamente dependendo da alimentação recebida pelo recém-nascido (Wallingford \& Underwood, 1986). A concentração de vitamina A no leite materno é suficiente para suprir as necessidades diárias supondo condições ideais de aleitamento. Contudo, caso o leite seja proveniente de nutrizes com dieta pobre em vitamina A, desnutridas, ou, caso a criança seja desmamada precocemente, as reservas continuarão baixas e aumentarão as probabilidades de desenvolvimento de xeroftalmia (OIson, 1987; WHO, 1996).

De forma fisiológica e natural, estão criadas as condições para que a criança, após o nascimento, pertença a um grupo de alto risco de carência de vitamina A. O presente estudo revela que essa hipótese está correta, mediante a avaliação do estado de vitamina A de recémnascidos em maternidades públicas no Rio de Janeiro, e serve para alertar os profissionais de saúde para a importância do controle e prevenção desse agravo nutricional nesse grupo populacional.

\section{Metodologia}

A população estudada foi constituída de 253 recém-nascidos, procedentes principalmente das zonas Norte e Oeste do Município do Rio de Janeiro, áreas consideradas de baixa condição sócio-econômica.

A coleta de dados ocorreu no período de dezembro de 1995 a maio de 1996, mediante dois plantões semanais de seis horas de dura- ção, o que correspondeu a oitenta e 173 casos, aproximadamente $13,9 \%$ e $10,6 \%$ do número total de partos ocorridos, no período de estudo, no Hospital Geral de Bonsucesso e Hospital Maternidade Carmela Dutra, respectivamente.

Após a coleta, as amostras de sangue foram submetidas à centrifugação (3.000 rpm) para separação e extração do soro, sendo imediatamente congeladas a uma temperatura de $-20 \circ \mathrm{C}$ nos bancos de sangue dos referidos locais de coleta. Uma vez por semana, as amostras foram transportadas para o Laboratório de Bioquímica do Instituto de Nutrição Josué de Castro, da Universidade Federal do Rio de Janeiro, para determinação espectrofotométrica dos níveis de retinol segundo o método Bessey-Lowrey modificado (Araújo \& Flores, 1978).

Os níveis de retinol sérico foram agrupa-

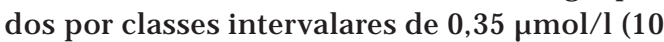
$\mu \mathrm{g} / \mathrm{dl}$ ) para permitir sua classificação de acordo com as recomendações da Organização Mundial da Saúde (WHO, 1996). Isso permite detectar os grupos com valores duvidosos $(0,70$ $\mu \mathrm{mol} / \mathrm{l}$ a $1,05 \mu \mathrm{mol} / \mathrm{l}$ ou $20 \mu \mathrm{g} / \mathrm{dl}$ a $30 \mu \mathrm{g} / \mathrm{dl}$ ), com carência marginal moderada $(0,35 \mu \mathrm{mol} / \mathrm{I}$ a $0,70 \mu \mathrm{mol} / \mathrm{l}$ ou $10 \mu \mathrm{g} / \mathrm{dl}$ a $20 \mu \mathrm{g} / \mathrm{dl}$ ) e com carência severa $(<0,35 \mu \mathrm{mol} / \mathrm{l}$ ou $<10 \mu \mathrm{g} / \mathrm{dl}$ ). No presente estudo, o ponto de corte de $<1,05$ $\mu \mathrm{mol} / \mathrm{l}(<30 \mu \mathrm{g} / \mathrm{dl}$ ) foi utilizado para indicar hipovitaminose.

Para caracterizar a amostra, real izaram-se entrevistas com as puerperas, obtendo-se, informações sobre renda familiar, escolaridade e condições de saneamento das moradias. A renda familiar foi expressa em salários mínimos (SM ) da época e categorizada em quatro grupos: menos de um salário mínimo (<1 SM); de um a dois salários mínimos ( 1 a 2 SM ); de três a cinco salários mínimos (3 a 5 SM) e mais de cinco salários mínimos (>5 SM).

A escolaridade das puerperas foi classificada como: analfabeta; primeiro grau incompleto; primeiro grau completo; segundo grau incompleto; segundo grau completo e terceiro grau completo. As condições de saneamento foram descritas segundo as informações de abastecimento de água (água ligada à rede pública com canalização; rede pública sem canalização; poço com e sem canalização e outras formas de abastecimento) e esgoto (ligado à rede geral; fossa e a céu aberto).

Adicionalmente, foram colhidas informações pertinentes ao concepto e à mãe: sexo do bebê, período gestacional, participação da mãe em programa de assistência pré-natal e tipo de parto. Dados complementares de interesse para a avaliação nutricional do recém-nascido, tais como: massa corporal e comprimento ao 
nascer, também foram coletados e comparados com os da população de referência americana, como sugerido pela Organização Mundial de Saúde (OMS, 1983). O cálculo dos valores $Z$ dos índices de massa corporal para idade (MC/I) e comprimento para idade $(\mathrm{C} / \mathrm{I})$ foi realizado por software desenvolvido pelo Centers for Disease Control and Prevention (CDC). Para ambos, o ponte de corte para desnutrição foi $Z \leq-2$. $O$ sobrepeso foi diagnosticado nas crianças com $\mathrm{MC} / \mathrm{I} \geq+2$.

Récem-nascidos com peso inferior a $2.500 \mathrm{~g}$ foram classificados como nascidos com baixo peso, e aqueles com período gestacional inferior a 37 semanas, como prematuros. O critério de nascimento a termo referiu-se a recém-nascidos com período gestacional entre 37 e 41 semanas, e considerou-se como participação em programa de assistência pré-natal a puerpera que realizou, no mínimo, uma consulta a cada trimestre.

A não-obtenção de todas as informações antropométricas foi motivada por intercorrências no momento do parto, quando al guns recém-nascidos necessitaram de cuidados especiais, o que impossibilitou a tomada de todas as medidas por ocasião da coleta de dados.

\section{Resultados}

Dos 253 recém-nascidos estudados, 58\% eram do sexo masculino, na sua maior parte pertencentes a famílias de baixa renda $55,8 \%$ com renda familiar até dois SM ), e 69,4\% apresentavam até o primeiro grau completo. A maior parte das resi dências tinha água $(56,1 \%)$, esgoto $(84,4 \%)$ ligado à rede pública e coleta de lixo regular (80,0\%). A mai oria das crianças (74,8\%) nasceu de parto normal, com acompanhamento pré-natal $(91,0 \%)$, apresentando freqüência média de cinco consultas. A prevalência de baixo peso ao nascer $(<2.500 \mathrm{~g}$ ) foi $12,6 \%$, e o percentual de prematuridade foi de $17,5 \%$ (Tabela 1 ).

A maioria $(55,7 \%)$ dos recém-nascidos apresentou valores de retinol sérico inferiores a 1,05 $\mu \mathrm{mol} / \mathrm{l}$, e 12,3\% dos valores ficaram abaixo de $0,35 \mu \mathrm{mol} / \mathrm{I}$ (Tabela 2). A concentração média de vitamina $A$ ( \pm desvio-padrão) no sangue de cordão umbilical foi $0,94 \pm 0,38 \mu \mathrm{mol} / \mathrm{l}$. A média para o sexo masculino foi $0,96 \pm 0,38$ $\mu \mathrm{mol} / \mathrm{l}$, essencialmente igual à do sexo feminino $(0,97 \pm 0,39 \mu \mathrm{mol} / \mathrm{l})$.

A prevalência de desnutrição $(Z \leq-2)$ considerando-se o índice massa corporal para idade foi $7,2 \%$, sendo bem mais freqüente em crianças do sexo masculino (10,4\%) do que em crian-
Tabela 1

Características gerais de 253 recém-nascidos assistidos na Maternidade Municipal Carmela Dutra e no Hospital Geral de Bonsucesso, no Município do Rio de J aneiro, 1995-1996.

\begin{tabular}{|c|c|c|}
\hline Variáveis & $\mathrm{n}$ & $\%$ \\
\hline \multicolumn{3}{|l|}{ Sexo } \\
\hline Masculino & 147 & 58,0 \\
\hline Feminino & 106 & 42,0 \\
\hline Total & 253 & 100,0 \\
\hline \multicolumn{3}{|l|}{ Tipo de parto } \\
\hline Normal & 181 & 74,8 \\
\hline Cesárea & 56 & 23,1 \\
\hline Pélvico & 1 & 0,4 \\
\hline Gemelar normal & 4 & 1,7 \\
\hline Total & 242 & 100,0 \\
\hline \multicolumn{3}{|l|}{ Pré-natal } \\
\hline Sim & 220 & 91,0 \\
\hline Não & 22 & 9,0 \\
\hline Total & 242 & 100,0 \\
\hline \multicolumn{3}{|l|}{ Peso ao nascer } \\
\hline$<2,500 \mathrm{~g}$ & 32 & 12,6 \\
\hline$\geq 2,500 \mathrm{~g}$ & 221 & 87,4 \\
\hline Total & 253 & 100,0 \\
\hline \multicolumn{3}{|l|}{ Idade gestacional } \\
\hline$<37$ semanas & 41 & 17,5 \\
\hline$\geq 37$ semanas & 205 & 82,5 \\
\hline Total & 234 & 100,0 \\
\hline \multicolumn{3}{|c|}{ Renda familiar (salários mínimos) } \\
\hline$>1$ & 22 & 8,8 \\
\hline 1 a 2 & 118 & 47,0 \\
\hline 3 a 5 & 67 & 26,7 \\
\hline$>5$ & 44 & 17,5 \\
\hline Total & 251 & 100,0 \\
\hline \multicolumn{3}{|c|}{ Escolaridade da puérpera } \\
\hline Analfabeta & 15 & 6,0 \\
\hline 1o grau incompleto & 61 & 24,5 \\
\hline 1o grau completo & 97 & 38,9 \\
\hline 2 o grau incompleto & 29 & 11,6 \\
\hline 2 o grau completo & 40 & 16,1 \\
\hline 3o grau completo & 7 & 2,9 \\
\hline Total & 249 & 100,0 \\
\hline
\end{tabular}




\section{Tabela 2}

Estado nutricional de vitamina A (níveis de retinol no sangue de cordão) de 253 recém-nascidos assistidos na Maternidade Municipal Carmela Dutra e no Hospital Geral de Bonsucesso, no Município do Rio de J aneiro, 1995-1996.

\begin{tabular}{lrc}
\hline $\begin{array}{l}\text { Estado nutricional de Vitamina A } \\
\text { (Retinol no sangue de cordão) }\end{array}$ & $\mathrm{n}$ & $\%$ \\
\hline$<0,35 \mu \mathrm{mol} / \mathrm{l}$ & 31 & 12,3 \\
$0,35 \vdash 0,70 \mu \mathrm{mol} / \mathrm{l}$ & 36 & 14,2 \\
$0,70 \vdash 1,05 \mu \mathrm{mol} / \mathrm{l}$ & 74 & 29,2 \\
$\geq 1,05 \mu \mathrm{mol} / \mathrm{l}$ & 112 & 44,3 \\
Total & 253 & 100,0 \\
\hline
\end{tabular}

ças do sexo feminino (2,8\%). O percentual de sobrepeso ( $Z \mathrm{MC} / \mathrm{I} \geq+2$ ) encontrado foi de 2,4\% (Tabela 3). Quando a desnutrição foi caracterizada segundo o índice comprimento para idade, o percentual encontrado foi de $3,6 \%$ (Tabela 3).

Dentre os recém-nascidos considerados desnutridos de acordo com o índice massa corporal para idade, $72,2 \%$ apresentaram hipovitaminose $\mathrm{A}$, enquanto dentre os eutróficos, a prevalência observada foi de $54,7 \%$. Quando a desnutrição foi caracterizada segundo comprimento para idade, $67,0 \%$ dos desnutridos apresentaram hipovitaminose A contra 55,5\% dos eutróficos (Tabela 4).

Os recém-nascidos com baixo peso ao nascer apresentaram tendência a uma maior prevalência de hipovitaminose $A(68,7 \%)$ do que aqueles com peso adequado (54,0\%) (Tabela 5). O percentual de hipovitaminose $A$ em recémnascidos prematuros e a termo foi de $51,2 \%$ e $56,3 \%$, respectivamente. A associação entre o peso ao nascer, o período gestacional e o estado nutricional de vitamina A revelou maior prevalência da hipovitaminose $A$ em recém-nascidos com baixo peso a termo $(77,0 \%)$, do que em recém-nascidos de baixo peso nascidos prematuramente $(62,2 \%)$ e de peso ao nascer adequado independente de prematuridade (Tabela 6).

\section{Discussão}

A epidemiologia da deficiência de vitamina A tem sido geralmente bem estudada em crianças de idade pré-escolar, entretanto as informações sobre recém-nascidos são mais escassas e ainda não se tem definido um padrão normal de concentração de retinol plasmático para esse grupo etário. Vários estudos sugerem a necessidade de uma atenção especial a este grupo populacional (Basu et al., 1994; Humphrey et al., 1996; Neel \& Alvarez, 1990; Rondo et al., 1995; Stoltzfus \& Underwood, 1995; Underwood, 1994).

O presente estudo mostra que os recémnascidos investigados em serviços públicos no Rio de Janeiro também merecem atenção especial com referência à hi povitaminose $\mathrm{A}$, apesar da crença generalizada de que essa carência específica estaria limitada às regiões mais pobres do País. Mais da metade do grupo estudado $(55,7 \%)$ apresentou hipovitaminose A definida bioquimicamente (WHO, 1996).

A baixa concentração de vitamina A sérica ao nascer deve-se à barreira natural (e necessária) imposta pela placenta (Basu et al., 1994). Essa idéia é apoiada por trabal hos experimentais que demonstram uma baixa reserva hepática de vitamina $A$ em ratos ao nascimento, apesar de a dieta ingerida durante a gestação garantir níveis adequados de vitamina A (Gardner \& Ross, 1993), e pelas observações de Wallingford \& Underwood (1986), segundo as quais recém-nascidos de mães bem-nutridas têm baixas concentrações de vitamina A hepática.

Basu et al. (1994) assinalam também como causa possível para essas reservas baixas ou inexistentes nos recém-nascidos a pequena produção de RBP (Retinol-binding protein), em virtude da imaturidade hepática ao nascer ( $60 \%$ dos valores observados nas respectivas mães), com uma saturação molar de vitamina A/ RBP próxima a um em ambos os grupos. Assim, os baixos valores de retinol observados em recém-nascidos podem estar mais relacionados a um reflexo próprio da idade do que a uma deficiência vitamínica verdadeira (Basu et al., 1994; Godel et al., 1996), havendo razões de sobra, portanto, para se acreditar que crianças menores são particularmente vulneráveis ao desenvolvimento de hipovitaminose A e dependem do aleitamento materno ou proveniente de outras fontes dietéticas para construir suas reservas (Humphrey et al., 1996).

Normalmente, a vitamina A é transferida da mãe para o filho sessenta vezes mais durante seis meses de lactação, ao se comparar com a acumulação feita pelo feto durante os nove meses de gestação. Todavia, a concentração de vitamina A no leite materno pode ser extremamente baixa em lactentes de países em desenvolvimento (Stoltzfus \& Underwood, 1995). Assim, um aporte satisfatório de vitamina A no período de lactação se reveste de grande importância a fim de garantir suprimento adequado à criança nos primeiros meses de vida, já que é natural esperar que a reserva do recém-nascido se construa após o nascimento. 
Tabela 3

Estado nutricional antropométrico, segundo escore $Z$ de massa corporal para idade (MC/I) e comprimento para idade (C/I), considerando-se o sexo de 253 recém-nascidos assistidos na Maternidade Municipal Carmela Dutra e no Hospital Geral de Bonsucesso, no Município do Rio de J aneiro, 1995-1996.

\begin{tabular}{|c|c|c|c|c|c|c|c|c|c|c|}
\hline \multirow[t]{4}{*}{ Sexo } & \multicolumn{10}{|c|}{ Estado nutricional antropométrico } \\
\hline & \multirow{2}{*}{\multicolumn{2}{|c|}{$\begin{array}{l}\text { Desnutrido } \\
(Z \leq-2)\end{array}$}} & \multicolumn{4}{|c|}{$\mathrm{MC} / \mathrm{I}$} & \multicolumn{4}{|c|}{$\mathrm{C} / \mathrm{l}$} \\
\hline & & & \multicolumn{2}{|c|}{$\begin{array}{c}\text { Eutrófico } \\
(-2<Z<+2)\end{array}$} & \multicolumn{2}{|c|}{$\begin{array}{l}\text { Sobrepeso } \\
(Z \geq+2)\end{array}$} & \multicolumn{2}{|c|}{$\begin{array}{l}\text { Desnutrido } \\
(Z \leq-2)\end{array}$} & \multicolumn{2}{|c|}{$\begin{array}{c}\text { Eutrófico } \\
(Z>-2)\end{array}$} \\
\hline & $\mathrm{n}$ & $\%$ & $n$ & $\%$ & $\mathrm{n}$ & $\%$ & $\mathrm{n}$ & $\%$ & $\mathrm{n}$ & $\%$ \\
\hline Feminino & 3 & 2,8 & 99 & 93,4 & 4 & 3,8 & 4 & 3,8 & 101 & 96,2 \\
\hline Masculino & 15 & 10,4 & 127 & 88,2 & 2 & 1,4 & 5 & 3,5 & 137 & 96,5 \\
\hline Total & 18 & 7,2 & 226 & 90,4 & 6 & 2,4 & 9 & 3,6 & 238 & 96,4 \\
\hline
\end{tabular}

\section{Tabela 4}

Relação entre o estado nutricional antropométrico ( $Z$ massa corporal para idade e comprimento para idade) e o estado nutricional de vitamina $A$ (níveis de retinol no sangue de cordão) de 253 recém-nascidos assistidos na Maternidade Municipal Carmela Dutra e no Hospital Geral de Bonsucesso, no Município do Rio de J aneiro, 1995-1996.

\begin{tabular}{|c|c|c|c|c|}
\hline \multirow[t]{3}{*}{ Estado nutricional antropométrico } & \multicolumn{4}{|c|}{ Estado nutricional de vitamina $A$} \\
\hline & \multicolumn{2}{|c|}{ Adequado $(\geq 1,05 \mu \mathrm{mol} / \mathrm{l})$} & \multicolumn{2}{|c|}{ Hipovitaminose $\mathrm{A}(<1,05 \mu \mathrm{mol} / \mathrm{l})$} \\
\hline & $\mathrm{n}$ & $\%$ & $n$ & $\%$ \\
\hline \multicolumn{5}{|l|}{ Massa corporal para idade } \\
\hline Desnutrido $(Z \leq-2)$ & 5 & 27,8 & 13 & 72,2 \\
\hline Eutrófico $(-2<Z<+2)$ & 102 & 45,3 & 123 & 54,7 \\
\hline Sobrepeso $(Z \geq+2)$ & 3 & 50,0 & 3 & 50,0 \\
\hline Total & 110 & 44,2 & 139 & 55,8 \\
\hline \multicolumn{5}{|l|}{ Comprimento para idade } \\
\hline Desnutrido $(Z \leq-2)$ & 3 & 33,0 & 6 & 67,0 \\
\hline Eutrófico $(Z>-2)$ & 106 & 44,5 & 132 & 55,5 \\
\hline Total & 109 & 44,1 & 138 & 55,9 \\
\hline
\end{tabular}

Tabela 5

Relação entre o estado nutricional de vitamina A (níveis de retinol no sangue de cordão) e peso ao nascer de 253 recém-nascidos assistidos na Maternidade Municipal Carmela Dutra e no Hospital Geral de Bonsucesso, no Município do Rio de J aneiro, 1995-1996.

\begin{tabular}{|c|c|c|c|c|}
\hline \multirow[t]{3}{*}{ Peso ao nascer } & \multicolumn{4}{|c|}{ Estado nutricional de vitamina $\mathrm{A}$} \\
\hline & \multicolumn{2}{|c|}{ Adequado $(\geq 1,05 \mu \mathrm{mol} / \mathrm{l})$} & \multicolumn{2}{|c|}{ Hipovitaminose A $(<1,05 \mu \mathrm{mol} / \mathrm{l})$} \\
\hline & $n$ & $\%$ & $n$ & $\%$ \\
\hline Baixo peso $(<2.500 \mathrm{~g})$ & 10 & 31,3 & 22 & 68,7 \\
\hline Peso adequado $(\geq 2.500 \mathrm{~g})$ & 102 & 46,0 & 119 & 54,0 \\
\hline Total & 112 & 44,3 & 141 & 55,7 \\
\hline
\end{tabular}


Tabela 6

Relação entre o estado nutricional de vitamina A (níveis de retinol no sangue de cordão), peso ao nascer e período gestacional de 253 recém-nascidos assistidos na Maternidade Municipal Carmela Dutra e no Hospital Geral de Bonsucesso, no Município do Rio de J aneiro, 1995-1996.

\begin{tabular}{|c|c|c|c|c|}
\hline \multirow[t]{3}{*}{ Peso ao nascer/Semana gestacional* } & \multicolumn{4}{|c|}{ Estado nutricional de vitamina $\mathrm{A}$} \\
\hline & \multicolumn{2}{|c|}{ Adequado $(\geq 1,05 \mu \mathrm{mol} / \mathrm{l})$} & \multicolumn{2}{|c|}{ Hipovitaminose $\mathrm{A}(<1,05 \mu \mathrm{mol} / \mathrm{l})$} \\
\hline & $n$ & $\%$ & $n$ & $\%$ \\
\hline Peso adequado/A termo & 78 & 43,4 & 102 & 56,6 \\
\hline Peso adequado/Prematuro & 12 & 48,0 & 13 & 52,0 \\
\hline Baixo peso/A termo & 3 & 23,0 & 10 & 77,0 \\
\hline Baixo peso/Prematuro & 6 & 37,5 & 10 & 62,5 \\
\hline
\end{tabular}

*Baixo peso (peso ao nascer $<2.500 \mathrm{~g}$ ) e prematuro ( $<37$ semanas).

Isso torna esse grupo populacional, entre os grupos de risco, o mais vulnerável aos efeitos deletérios da carência marginal de vitamina $A$, principalmente pelo rápido crescimento que as crianças experimentam nos primeiros meses de vida.

Considerando que não há motivos para esperar reserva hepática de vitamina A em recém-nascidos, não é de estranhar que não se tenham observado grandes diferenças na prevalência de hipovitaminose $A$ no sangue de cordão entre prematuros e recém-nascidos a termo, tanto de peso adequado (48,0\% e 43,4\%, respectivamente), quanto de baixo peso $(37,5 \%$ e $23 \%$, respectivamente). Vários autores recomendam especial atenção a recém-nascidos prematuros, pela tendência de estes apresentarem uma menor reserva de retinol hepático ao nascimento (Brandt et al., 1978; Shenai et al., 1981; Stoltzfus \& Underwood, 1995; Underwood, 1996). No entanto, tal recomendação, apesar de lógica, não teria tradução prática, desde que os recém-nascidos normais já mereceriam o máximo de atenção nas condições que determinam a construção de suas reservas hepáticas.

Os resultados do presente estudo mostram que a prevalência de hipovitaminose $A$ nos recém-nascidos é comparável às cifras que se encontram nas regiões mais pobres do mundo, sem que isso pareça ter relação com o período gestacional ou com o grau de distrofia da criança. A hipovitaminose $A$ foi igualmente elevada em desnutridos e eutróficos, independentemente do índice utilizado para a classificação do estado nutricional, ainda que tenha sido observada uma tendência a prevalências maiores em desnutridos (Tabela 4). Não cabe aqui discutir o significado das diferenças encontradas entre eutróficos e desnutridos, já que o peso dessa observação recai sobre o fato de que crianças eutróficas também apresentam uma alta prevalência de hipovitaminose $A$.

O estabelecimento de um padrão de concentração de retinol plasmático (e hepático) em recém-nascidos é de extrema importância em termos de saúde preventiva, em razão da grave repercussão da hipovitaminose A nas taxas de morbi-mortalidade infantil.

A condição de alto risco para o desenvolvimento de carência de vitamina A após o nascimento parece justificar a ampliação do diagnóstico do estado nutricional de vitamina A no Município do Rio de Janeiro, o que se somaria às informações já coletadas em outras regiões do País, levando a uma reflexão sobre a pertinência da deflagração de um programa de intervenção nutricional. Tal fato melhoraria o estado nutricional de vitamina $A$ do grupo em questão, em conjunto com as demais ações básicas em prol da saúde e sobrevivência infantil. 


\section{Referências}

ARAÚJO, C. R. C. \& FLORES, H., 1978. Improved spectrophotometric vitamin A assay. Clinical Chemistry, 24:386

BASU, T. K.; WEIN, E. E.; GANGOPADHYAY, K. C.; WOLEVER, T. M. S. \& GODEL, J. C., 1994. Plasma vitamin $A$ (retinol) and retinol-binding protein in newborns and their mothers. Nutrition Research, 14:297-1.303.

BRANDT, R. B.; MUELLER, D. G.; SCHROEDER, J. R. \& GUYER, K. E., 1978. Journal of Pediatrics, 92:101104.

GARDNER, E. M. \& ROSS, A. C., 1993. Dietary vitamin A restriction produces marginal vitamin A status in young rats. Journal of Nutrition, 123:1.4351.443.

GEBRE-MEDHIN, M. \&VAHLQUIST, A., 1984. Vitamin $A$ in the human foetus. Acta Pediatrica Scandinavica, 73:333-340.

GODEL, J. C.; BASU, T. K.; PABST, H. F.; HODGES, S. R.; HODGES, P. E. \& M ARGARET, L. N., 1996. Perinatal vitamin $A$ (retinol) status of northern Canadian mothers and their infants. Biology of the Neonate, 69:133-139.

HUMPHREY, J. H.; AGOESTINA, T.; WU, L.; USMAN, A.; NURACHIN, M.; SUBARDJA, D.; HIDAYAT, S.; TIELSCH, J.; WEST, K. P. \& SOM MER, A., 1996. Impact of neonatal vitamin A supplementation on infant morbidity and mortality. Journal of Pediatrics, 128:489-496.

NEEL, N. R. \& ALVAREZ, J., 1990. Chronic fetal malnutrition and vitamin $A$ in cord serum. European Journal of Clinical Nutrition, 44:207-212.

OLSON, J. A., 1987. Recommended dietary intakes (RDI) of vitamin in humans. American Journal of Clinical Nutrition, 45:704-716.
OMS (Organización Mundial de la Salud), 1983. Medición del Cambio del Estado Nutricional. Ginebra: OMS.

RONDO, P. H. C.; ABBOTT, R.; RODRIGUES, L. C. \& TOM KINS, A. M., 1995. Vitamin A, folate, and iron concentrations in cord and maternal blood of intra-uterine growth retarded and appropriate birth weight babies. European Journal of Clinical Nutrition, 49:391-399.

SHENAI, J. P.; CHYTIL, F. \& STAHLM AN, M. T., 1981. Plasma vitamin $A$ and retinol-binding protein in premature and term neonates. Journal of Pediatrics, 99:302-305.

STOLTZFUS, R. J. \& UNDERWOOD, B. A., 1995. Breast-milk vitamin $A$ as an indicator of the vitamin A status of women and infants. Bulletin of the World Health Organization, 73:703-711.

UNDERWOOD, B. A., 1994. Maternal vitamin A status and its importance in infancy and early chilhood. American Journal of Clinical Nutrition, 59(Sup.): 517s-524s.

WALLINGFORD, J. C. \& UNDERWOOD, B. A., 1986. Vitamin A deficiency in pregnancy, lactation, and the nursing child. In: Vitamin A: Deficiency and its Control (J. C. Bauernfeind, ed.), pp. 101-152, New York: Academic Press.

WHO (World Health Organization), 1996. Indicators for Assessing Vitamin A Deficiency and their Application in Monitoring and Evaluating Intervention Programmes (Micronutrient Series, 10). Geneva: WHO. 